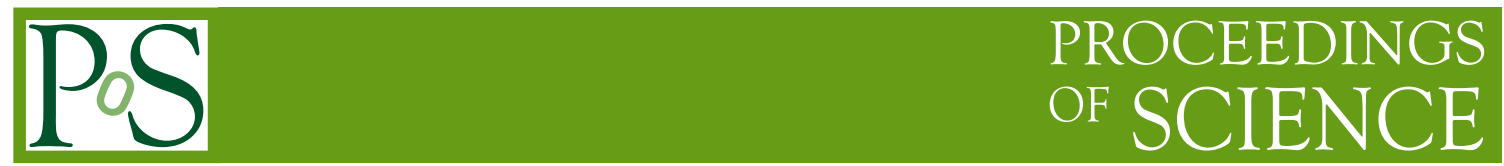

\title{
Electroweak corrections in top physics (2)
}

\section{Davide Pagani*}

Centre for Cosmology, Particle Physics and Phenomenology (CP3), Université Catholique de Louvain, B-1348 Louvain-la-Neuve, Belgium

E-mail: davide.pagani@uclouvain.be

In this proceeding we review the calculations of electroweak corrections in top-quark physics. We discuss the impact of electroweak corrections to the top-quark charge asymmetry and to the cross sections of various subdominant top-quark production mechanisms at hadron colliders, namely, single-top production channels and the associate productions of a top-quark pair with a heavy boson.

8th International Workshop on Top Quark Physics, TOP2015

14-18 September, 2015

Ischia, Italy

${ }^{*}$ Speaker. 
In this proceeding we review the calculations of electroweak corrections in top-quark physics at hadron colliders. The impact of electroweak corrections to the cross section of top-quark pair production is considered in another contribution of this conference proceedings [1] and has also been reviewed in [2].

\section{Top-quark charge asymmetry}

At the Tevatron, since the initial state is invariant under CP transformation, the top-quark charge asymmetry is equivalent to a forward-backward asymmetry. This observable has received particular attention in the recent years due to the observed deviations between Standard Model (SM) predictions and measured values, which reached up to $3.5 \sigma$ in the analysis presented in [3] by the CDF collaboration. At the LHC, a forward-backward asymmetry is not present, since proton-proton collisions are symmetric, but it is possible to measure a so-called central asymmetry, which has the same physics origin. In the following we will refer only to the forward-backward asymmetry, however, the same arguments apply also to the central asymmetry.

At LO, which is of $\mathscr{O}\left(\alpha_{s}^{2}\right)$, the top-quark pair hadroproduction originates from $q \bar{q}$ and $g g$ initial states via QCD interactions. However, LO contributions are symmetric. QCD induces asymmetric terms only starting from NLO corrections [4], which are of $\mathscr{O}\left(\alpha_{s}^{3}\right)$. The forward-backward asymmetry, independently form the specific definition employed, can be in general written as

$$
A_{t \bar{t}}=\frac{\sigma_{+}-\sigma_{-}}{\sigma_{+}+\sigma_{-}}
$$

The numerator of the r.h.s of eq. (1.1) consists of the difference between the cross sections for the production of a top-quark respectively in the forward and backward direction, while the denominator is the total cross section. Thus, by expanding $A_{t \bar{t}}$ in powers of $\alpha_{s}$ we obtain that the LO prediction for $A_{t \bar{t}}$ is of $\mathscr{O}\left(\alpha_{s}\right)$, i.e., it is induced by the asymmetric NLO QCD corrections of $\mathscr{O}\left(\alpha_{s}^{3}\right)$ to the cross section.

Electroweak interactions induce non-vanishing asymmetric terms to the numerator of $A_{t \bar{t}}$ (see ref. [5]) starting from contributions of $\mathscr{O}\left(\alpha_{s}^{2} \alpha\right)$ and $\mathscr{O}\left(\alpha^{2}\right)$. The former consists of NLO EW corrections to the cross section and leads to $\mathscr{O}(\alpha)$ contributions to $A_{t \bar{t}}$, while the latter originates form tree-level only diagrams and lead to $\mathscr{O}\left(\alpha^{2} / \alpha_{s}^{2}\right)$ contributions to $A_{t \bar{t}}$.

Independently from the perturbative order considered, since the $g g$ initial state is symmetric, it cannot contribute to the asymmetry. Thus the asymmetric terms from the $\mathscr{O}\left(\alpha_{s}^{3}\right), \mathscr{O}\left(\alpha_{s}^{2} \alpha\right)$ and $\mathscr{O}\left(\alpha^{2}\right)$ contributions can originate from the $q \bar{q}$ initial state but not from $g g$. In particular, the NLO QCD $\mathscr{O}\left(\alpha_{s}^{3}\right)$ asymmetric terms originate only from the interference of tree-level diagrams and boxes and, equivalently, only from the interference of the real gluon emissions from the initial and final state. ${ }^{1}$ For this reason it is useful to divide the NLO EW $\mathscr{O}\left(\alpha_{s}^{2} \alpha\right)$ asymmetric terms into NLO QED contributions, which involve only QCD and QED interactions, and NLO Weak contributions involving the remaining weak interactions. Indeed, the asymmetric NLO QED contributions can be obtained by substituting in all the possible way one gluon into one photon in the matrix elements entering the calculation of the asymmetric NLO QCD contributions. Thus, the NLO QED

\footnotetext{
${ }^{1}$ Also (anti)quark radiation via the $q g(\bar{q} g)$ initial state induces an asymmetry. However, its size is negligible and thus not discussed here.
} 
contributions can be simply obtained by multiplying the NLO QCD contribution by the $R_{Q E D}\left(Q_{q}\right)$ factor defined as

$$
R_{Q E D}\left(Q_{q}\right)=\frac{36}{5} Q_{q} Q_{t} \frac{\alpha}{\alpha_{s}} \Longrightarrow \quad R_{Q E D}\left(Q_{u}\right) \sim 0.2, \quad R_{Q E D}\left(Q_{d}\right) \sim-0.1
$$

where $Q_{q}$ is the charge of the quark in the initial state and the numerical factor is due to color. This result is independent of the specific definition used for the asymmetry and the cuts applied on the $t \bar{t}$ pair.

The NLO Weak contributions originate from the same diagrams of the NLO QED ones, where the photon has to be replaced by a $Z$ boson. ${ }^{2}$ In this case the value cannot be expressed via a simple factor that is independent on the cuts and the specific definition of $A_{t \bar{t}}$.

The $\mathscr{O}\left(\alpha^{2}\right)$ contributions simply originate from tree-level diagrams with only EW interactions, i.e., from the $s$-channel production via a photon or a $Z$ boson.

The total size of the EW $\mathscr{O}(\alpha)+\mathscr{O}\left(\alpha^{2} / \alpha_{s}^{2}\right)$ contributions to $A_{t \bar{t}}$ amounts to $\sim 20 \%$ of the $\mathscr{O}\left(\alpha_{s}\right)$ contribution from pure QCD effects, the precise value of this quantity depends on the specific definition of $A_{t \bar{t}}$, the cuts and the input parameters. On the contrary, the dominant component of the electroweak contributions, the QED part of the $\mathscr{O}(\alpha)$, is independent of the cuts applied, the $A_{t \bar{t}}$ definition and, obviously, the QCD renormalisation scale.

The electroweak contributions to the asymmetry, being $\sim 20 \%$ of the QCD-induced one and with the same overall sign, decrease the deviation between the SM theoretical predictions and the values measured by the CDF and D0 collaborations. Very recently also the NNLO QCD corrections of $\mathscr{O}\left(\alpha_{s}^{4}\right)$ to the numerator of $A_{t \bar{t}}$ have been calculated [6] and, together with the new results from analyses based on larger data sets, they have further contributed to diminish the previously observed deviations. Moreover, this calculation has a posteriori proved that the approach consisting in a pure expansion in powers of $\alpha_{s}$ and $\alpha$ for $A_{t \bar{t}}$, as presented here, is preferable to the inclusion of $\mathscr{O}\left(\alpha_{s}^{3}\right)$ terms in the denominator of $A_{t \bar{t}}$ without the $\mathscr{O}\left(\alpha_{s}^{4}\right)$ terms in the numerator. Indeed both contributions enter the $\mathscr{O}\left(\alpha_{s}^{2}\right)$ term of $A_{t \bar{t}}$, but they have opposite signs and largely cancel each other. As said, the same physics can be studied at the LHC via a central asymmetry. In this case the relative size of the EW contributions is a bit smaller, i.e., $\sim 15-20 \%$ of the QCD-induced term, depending on the proton-proton c.o.m. energy (see for further details Refs. [7, 8]). In the case of the central asymmetry at the LHC, no significant deviation between SM predictions and measured values is present at the moment.

Besides EW fixed-order contributions, effects from resummed EW Sudakov logarithms have also been studied in [9] and found to be $\sim 5 \%$ of the QCD-induced $\mathscr{O}\left(\alpha_{s}\right)$ contribution of $A_{t \bar{t}}$. However, this result cannot be straightforwardly added to the previously discussed fixed-order contributions, because it would lead to a non-negligible double counting of $\mathscr{O}(\alpha)$ Sudakov logarithms.

\section{Top-quark pair production in association with a heavy boson}

The production of a top-quark pair in association with a heavy boson consists of the following different final states: $t \bar{t} H, t \bar{t} Z, t \bar{t} W^{+}$and $t \bar{t} W^{-}$. All these processes are relevant in the context of SM

\footnotetext{
${ }^{2}$ Although it can be formally included, the $Z$ boson real radiation ( $\left.t \bar{t} Z\right)$ is typically discarded, since it leads to negligible contributions.
} 


\begin{tabular}{c|cc}
$13 \mathrm{TeV}: \delta(\%)$ & $t \bar{t} H$ & $t \bar{t} Z$ \\
\hline NLO QCD & $29.7_{-11.1}^{+6.8} \pm 2.8\left(24.2_{-10.6}^{+4.8} \pm 4.5\right)$ & $45.9_{-15.5}^{+13.2} \pm 2.9\left(40.2_{-15.0}^{+11.1} \pm 4.7\right)$ \\
\hline LO EW & $1.2 \pm 0.9(2.8 \pm 2.0)$ & $0.0 \pm 0.7(2.1 \pm 1.6)$ \\
LO EW no $\gamma$ & $-0.4 \pm 0.0(-0.2 \pm 0.0)$ & $-1.1 \pm 0.0(-0.3 \pm 0.0)$ \\
\hline NLO EW & $-1.2 \pm 0.1(-8.2 \pm 0.3)$ & $-3.8 \pm 0.2(-11.1 \pm 0.5)$ \\
NLO EW no $\gamma$ & $-1.4 \pm 0.0(-8.5 \pm 0.2)$ & $-4.1 \pm 0.1(-11.5 \pm 0.3)$ \\
\hline HBR & $0.89(1.87)$ & $0.96(2.13)$
\end{tabular}

Table 1: Relative corrections for $t \bar{t} H$ and $t \bar{t} Z$ production in the $\alpha\left(m_{Z}\right)$-scheme at $13 \mathrm{TeV}$. The errors correspond to PDF uncertainties, for the NLO QCD also the scale uncertainties are displayed.

\begin{tabular}{c|cc}
$13 \mathrm{TeV}: \delta(\%)$ & $t \bar{t} W^{+}$ & $t \bar{t} W^{-}$ \\
\hline NLO QCD & $50.1_{-13.5}^{+14.2} \pm 2.4\left(59.7_{-17.7}^{+18.9} \pm 3.1\right)$ & $51.5_{-13.8}^{+14.8} \pm 2.8\left(66.3_{-19.6}^{+21.7} \pm 3.9\right)$ \\
\hline LO EW & 0 & 0 \\
LO EW no $\gamma$ & 0 & 0 \\
\hline NLO EW & $-7.7 \pm 0.2(-19.2 \pm 0.7)$ & $-6.7 \pm 0.2(-18.3 \pm 0.8)$ \\
NLO EW no $\gamma$ & $-8.0 \pm 0.2(-20.0 \pm 0.5)$ & $-7.0 \pm 0.2(-19.1 \pm 0.6)$ \\
\hline HBR & $3.88(7.41)$ & $6.50(15.01)$
\end{tabular}

Table 2: Same as in Table 1, but for $t \bar{t} W^{+}$and $t \bar{t} W^{-}$

and Beyond-the-Standard-Model (BSM) both as background and signal. For instance, the measurements of $t \bar{t} H$ and $t \bar{t} Z$ production can be respectively used to directly probe the coupling of the top quark with the Higgs and $Z$ boson. On the other hand, $t \bar{t} Z$ and $t \bar{t} W^{ \pm}$can be irreducible backgrounds of $t \bar{t} H$ production. Thus, precise predictions for these processes are extremely important.

NLO QCD calculations to the production of a top-quark pair in association with a heavy boson have been computed for the first time in $[10,11,12,13,14,15,16,17,18,19,20]$. These corrections have been recently (re-)calculated in [21] for all these processes and also for the associate production of a top-quark pair with two vector bosons and for four-top production. In [21] NLO QCD corrections to several distributions and at different hadronic c.o.m. energies are shown and their dependence on renormalisation and factorisation scale definitions is discussed in detail. In the following we focus on effects from electroweak corrections. NLO Weak corrections to $t \bar{t} H$ production have been calculated in [22] with a semi-automatic approach and including also NLO QED effects in [23]. Afterwards, NLO EW (Weak+QED) corrections have been calculated in a completely automated approach in [24] for the $t \bar{t} H, t \bar{t} Z, t \bar{t} W^{+}$and $t \bar{t} W^{-}$processes, together with NLO QCD corrections. We will discuss these results from [24], which have been calculated within the same framework (MADGRAPH5_AMC@NLO [25]) and using the same input parameters.

The LO cross section for the associate production of a top-quark pair with a heavy boson is of $\mathscr{O}\left(\alpha_{s}^{2} \alpha\right)$. In [24] the NLO QCD corrections of $\mathscr{O}\left(\alpha_{s}^{3} \alpha\right)$, the NLO EW corrections of $\mathscr{O}\left(\alpha_{s}^{2} \alpha^{2}\right)$ and the $\mathscr{O}\left(\alpha_{s} \alpha^{2}\right)$ contribution from only tree-level diagrams, the so-called LO EW contribution, have been calculated. The EW corrections (LO EW and NLO EW) have been presented both with and without the contribution from initial-state photons, which has been evaluated via the NNPDF2.3_QED PDF set [26] including the photon PDF. We have summarised inclusive results at $13 \mathrm{TeV}$ in Table 1 for $t \bar{t} H$ and $t \bar{t} Z$ and in Table 2 for $t \bar{t} W^{+}$and $t \bar{t} W^{-}$. Results in brackets refers to 
the boosted regime where a cut of $200 \mathrm{GeV}$ on the transverse momenta of the top, the antitop and the heavy boson is applied. These kind of cuts are particularly interesting for the case of $t \bar{t} H$, since it was shown in [27] that the signal over background ratio could be enhanced with boosted Higgs boson and top quarks. As expected, due to the Sudakov logarithms, NLO EW corrections are in this regime larger in magnitude and negative.

For $t \bar{t} H$ and $t \bar{t} Z$ production, electroweak corrections to the total cross-section are within the NLO QCD scale uncertainties, whereas in the boosted regime or in general for large $p_{t}$ 's they are comparable in size with scale uncertainties and thus very important. Relatively to the size of the corrections, the PDF error is large and mostly induced by the initial-state photons in the LO EW. The situation for $t \bar{t} W^{+}$and $t \bar{t} W^{-}$is analogous, but corrections are much larger and LO EW contributions are analytically zero. Also photon PDF errors are relatively small. In both Tables 1 and 2 the contribution from the radiation of an additional heavy boson (HBR), which is of $\mathscr{O}\left(\alpha_{s}^{2} \alpha^{2}\right)$ as the NLO EW, is also listed. Depending on the specific experimental analyses, this final state may contribute to the inclusive associate production of a top quark pair with a heavy boson, leading to partial cancellations of the EW Sudakov logarithms or, in the case of $t \bar{t} W^{ \pm}$even to overcompensations (see [24] for details).

\section{Single top}

Single top production can be classified in three classes of processes: $s$-channel, $t$-channel and associate $t W$ production. Again, we focus only on EW corrections. An updated list of references of calculations of QCD corrections can be found in the recent review in [28], which covers several aspects of top-quark physics at colliders.

The calculation of NLO EW corrections of the associated $t W$ production has been performed firstly without taking into account hard real-photon radiation in [29] and then by including it in [30]. Both the calculations have been performed for the extended MSSM NLO EW corrections, explicitly separating the SM subset from the contribution induced by loops with supersymmetric particles. Similarly, the calculation of NLO EW corrections to the $t$-channel production has been performed firstly without hard radiation in [31] and then by including it in [32], again within the MSSM and separating the SM-subset contribution. The partonic NLO EW corrections to $s$ - and $t$-channel production have been calculated also in [33] and integrated in the Monte-Carlo tool MCSANC-V1.01 [34]. All these calculations have been performed in the five-flavour scheme. In the following we will comment results for the LHC with $14 \mathrm{TeV}$ c.o.m. energy from [30, 32, 34].

In $t W$ associate production, NLO EW corrections to the $m(t W)$ distribution range from $+5 \%$ of the LO prediction, at the threshold, to $-3 \%$ at $m(t W)=1.3 \mathrm{TeV}$. In $t$-channel production NLO EW corrections to the total cross section are $\sim-3.6 \%$ of the LO, i.e., almost of the same absolute size of NLO QCD corrections $(\sim+4 \%)$. However, they grow in magnitude for large $p_{T}(t)$, e.g., they reach the value of $-15 \%$ at $p_{T}(t)=500 \mathrm{GeV}$. In $s$-channel production, NLO EW corrections are $\sim-2 \%$ of the LO prediction.

All these results do not include contributions form initial-state photons, which may be not negligible and potentially introduce large uncertainties due to the photon PDF. However, similarly to the case of NLO QCD, photon-initiated quark radiation would introduce, e.g., $t \bar{t}$ resonant configurations, which have to be removed or subtracted. 


\section{Conclusion}

In this proceeding we have discussed the impact of EW corrections in top-quark physics. Specifically, we focused on the case of the top-quark charge asymmetry, the associate production with a heavy boson and the single-top production mechanisms. The size of the corrections is in general not negligible, especially for the case of the charge asymmetry and in the tail of the distributions in single-top and associate production.

\section{References}

[1] P. Uwer, Electroweak corrections in top physics (1), in 8th International Workshop on Top Quark Physics, TOP2015 14-18 September, 2015, Ischia, Italy.

[2] D. Pagani, The electroweak contribution to top quark pair production: cross sections and asymmetries, in 7th Workshop on the CKM Unitarity Triangle (CKM 2012) Cincinnati, Ohio, USA, September 28-October 2, 2012, 2012. arXiv:1212.0808.

[3] CDF Collaboration, T. Aaltonen et al., Evidence for a Mass Dependent Forward-Backward Asymmetry in Top Quark Pair Production, Phys. Rev. D83 (2011) 112003, [arXiv: 1101.0034 ].

[4] J. H. Kuhn and G. Rodrigo, Charge asymmetry of heavy quarks at hadron colliders, Phys.Rev. D59 (1999) 054017, [hep-ph/9807420].

[5] W. Hollik and D. Pagani, The electroweak contribution to the top quark forward-backward asymmetry at the Tevatron, Phys.Rev. D84 (2011) 093003, [arXiv:1107.2606].

[6] M. Czakon, P. Fiedler, and A. Mitov, Resolving the Tevatron Top Quark Forward-Backward Asymmetry Puzzle: Fully Differential Next-to-Next-to-Leading-Order Calculation, Phys. Rev. Lett. 115 (2015), no. 5 052001, [arXiv:1411.3007].

[7] J. H. Kuhn and G. Rodrigo, Charge asymmetries of top quarks at hadron colliders revisited, JHEP 01 (2012) 063, [arXiv:1109.6830].

[8] W. Bernreuther and Z.-G. Si, Top quark and leptonic charge asymmetries for the Tevatron and LHC, Phys. Rev. D86 (2012) 034026, [arXiv: 1205 . 6580].

[9] A. V. Manohar and M. Trott, Electroweak Sudakov Corrections and the Top Quark Forward-Backward Asymmetry, Phys. Lett. B711 (2012) 313-316, [arXiv: 1201.3926 ].

[10] W. Beenakker, S. Dittmaier, M. Kramer, B. Plumper, M. Spira, et al., Higgs radiation off top quarks at the Tevatron and the LHC, Phys.Rev.Lett. 87 (2001) 201805, [hep-ph/ 0107081 ].

[11] W. Beenakker, S. Dittmaier, M. Kramer, B. Plumper, M. Spira, et al., NLO QCD corrections to $t$ anti-t H production in hadron collisions, Nucl.Phys. B653 (2003) 151-203, [hep-ph/0211352].

[12] S. Dawson, L. Orr, L. Reina, and D. Wackeroth, Associated top quark Higgs boson production at the LHC, Phys.Rev. D67 (2003) 071503, [hep-ph/0211438].

[13] S. Dawson, C. Jackson, L. Orr, L. Reina, and D. Wackeroth, Associated Higgs production with top quarks at the large hadron collider: NLO QCD corrections, Phys.Rev. D68 (2003) 034022, [hep-ph/0305087].

[14] V. Hirschi, R. Frederix, S. Frixione, M. V. Garzelli, F. Maltoni, et al., Automation of one-loop QCD corrections, JHEP 1105 (2011) 044, [arXiv:1103.0621]. 
[15] A. Lazopoulos, T. McElmurry, K. Melnikov, and F. Petriello, Next-to-leading order QCD corrections to $t \bar{t} Z$ production at the LHC, Phys.Lett. B666 (2008) 62-65, [arXiv : 0804 . 2220].

[16] M. Garzelli, A. Kardos, C. Papadopoulos, and Z. Trocsanyi, Z0 - boson production in association with a top anti-top pair at NLO accuracy with parton shower effects, Phys.Rev. D85 (2012) 074022 , [arXiv:1111.1444].

[17] A. Kardos, Z. Trocsanyi, and C. Papadopoulos, Top quark pair production in association with a Z-boson at NLO accuracy, Phys.Rev. D85 (2012) 054015, [arXiv:1111. 0610 ].

[18] M. Garzelli, A. Kardos, C. Papadopoulos, and Z. Trocsanyi, $t \bar{t} W^{+-}$and $t \bar{t} Z$ Hadroproduction at NLO accuracy in QCD with Parton Shower and Hadronization effects, JHEP 1211 (2012) 056, [arXiv:1208.2665].

[19] J. M. Campbell and R. K. Ellis, $t \bar{t} W^{+-}$production and decay at NLO, JHEP 1207 (2012) 052, [arXiv:1204.5678].

[20] F. Maltoni, M. Mangano, I. Tsinikos, and M. Zaro, Top-quark charge asymmetry and polarization in $t \bar{t} W$ production at the LHC, Phys.Lett. B736 (2014) 252-260, [arXiv: 1406.3262 ].

[21] F. Maltoni, D. Pagani, and I. Tsinikos, Associated production of a top-quark pair with vector bosons

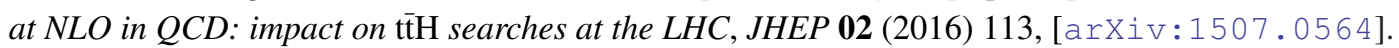

[22] S. Frixione, V. Hirschi, D. Pagani, H. Shao, and M. Zaro, Weak corrections to Higgs hadroproduction in association with a top-quark pair, JHEP 1409 (2014) 065, [arXiv: 1407 . 0823].

[23] Y. Zhang, W.-G. Ma, R.-Y. Zhang, C. Chen, and L. Guo, QCD NLO and EW NLO corrections to t $\bar{t} H$ production with top quark decays at hadron collider, Phys.Lett. B738 (2014) 1-5,

[arXiv:1407.1110].

[24] S. Frixione, V. Hirschi, D. Pagani, H. S. Shao, and M. Zaro, Electroweak and QCD corrections to top-pair hadroproduction in association with heavy bosons, JHEP 06 (2015) 184, [arXiv:1504.0344].

[25] J. Alwall, R. Frederix, S. Frixione, V. Hirschi, F. Maltoni, et al., The automated computation of tree-level and next-to-leading order differential cross sections, and their matching to parton shower simulations, JHEP 1407 (2014) 079, [arXiv: 1405.0301 ].

[26] NNPDF Collaboration, R. D. Ball, V. Bertone, S. Carrazza, L. Del Debbio, S. Forte, A. Guffanti, N. P. Hartland, and J. Rojo, Parton distributions with QED corrections, Nucl. Phys. B877 (2013) 290-320, [arXiv:1308.0598].

[27] T. Plehn, G. P. Salam, and M. Spannowsky, Fat Jets for a Light Higgs, Phys.Rev.Lett. 104 (2010) 111801, [arXiv:0910.5472].

[28] V. del Duca and E. Laenen, Top physics at the LHC, arXiv:1510.0669.

[29] M. Beccaria, G. Macorini, F. M. Renard, and C. Verzegnassi, Associated tW production at lhc: A Complete calculation of electroweak supersymmetric effects at one loop, Phys. Rev. D73 (2006) 093001, [hep-ph/0601175].

[30] M. Beccaria, C. M. Carloni Calame, G. Macorini, G. Montagna, F. Piccinini, F. M. Renard, and C. Verzegnassi, A Complete one-loop description of associated $t W$ production at LHC and a search for possible genuine supersymmetric effects, Eur. Phys. J. C53 (2008) 257-265, [arXiv:0705.3101].

[31] M. Beccaria, G. Macorini, F. M. Renard, and C. Verzegnassi, Single top production in the t-channel at LHC: A Realistic test of electroweak models, Phys. Rev. D74 (2006) 013008, [hep-ph/ 0605108 ]. 
[32] E. Mirabella, Electroweak Corrections to t-channel single top production at the LHC, Nuovo Cim. B123 (2008) 1111-1117, [arXiv:0811.2051].

[33] D. Bardin, S. Bondarenko, L. Kalinovskaya, V. Kolesnikov, and W. von Schlippe, Electroweak Radiative Corrections to Single-top Production, Eur. Phys. J. C71 (2011) 1533, [arXiv:1008.1859].

[34] S. G. Bondarenko and A. A. Sapronov, NLO EW and QCD proton-proton cross section calculations with mcsanc-v1.01, Comput. Phys. Commun. 184 (2013) 2343-2350, [arXiv:1301.3687]. 